\section{Entretien avec Frédéric Worms}

Philosophe et professeur

de philosophie

contemporaine à l'École

normale supérieure,

membre du Comité

consultatif national

d'éthique pour les

sciences de la vie

et de la santé

Marie Gaille ${ }^{1}$, Jean-Luc Teillaud ${ }^{2}$ médecine/sciences. Vous êtes membre du Comité consultatif national d'éthique pour les sciences de la vie et de la santé (CCNE) depuis 2013. Avant d'aller plus avant, pouvez-vous définir ce qu'est à ce jour la bioéthique pour un philosophe? Lors d'une conférence du CCNE, vous avez dit que "nous entrions dans un deuxième âge de la bioéthique, caractérisé par les relations entre acteurs, tandis que le premier aménageait la coexistence des valeurs et des cultures ».

Frédéric Worms. Cela découle d'une réflexion générale sur ce qui constitue la bioéthique, réflexion qui est l'objet de beaucoup de malentendus. Or, on peut dire que la bioéthique n'est pas une éthique, au sens où ce n'est pas un système général de morale, élaboré d'un coup par qui que ce soit, même par l'État, surtout pas par l'État. L'État n'est pas chargé de dispenser une éthique. La bioéthique est plutôt une démarche par laquelle on aborde les contradictions éthiques suscitées par les démarches biologiques et médicales. $\varepsilon t$ je dis bien des contradictions, c'est-à-dire des contradictions entre des éthiques, et pas seulement des contradictions entre quelque chose d'éthique et quelque chose qui serait une violence, ou contraire à l'éthique. Je pense que c'est extrêmement important de voir que c'est une éthique de second degré. II est également très important de dire que la bioéthique ne se réduit pas à un ensemble de procédures. Car le risque, quand on décrit les choses ainsi, c'est de dire que la bioéthique est un cadre de discussion entre des positions qui sont contradictoires. Si c'est seulement un cadre, il n'y a pas de contenu. Or, on est quand même obligé d'avoir des décisions sur le contenu. C'est aussi pour cela que la bioéthique requiert à la fois des connaissances factuelles - et c'est pour cela qu'on a besoin des médecins et des savants - et des confrontations entre des principes et des enjeux sociaux. C'est pour cela que la bioéthique est nécessairement pluridisciplinaire.

Cet entretien a été réalisé les 11 et 25 octobre 2018 et retranscrit par Marie Gaille. Vignette (Photo @ Inserm -Patrick Delapierre).

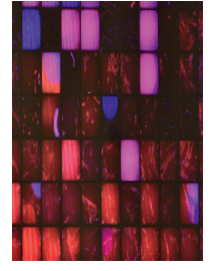

Mais si je reviens à votre question, il m'a semblé que la bioéthique consiste à dépasser, non pas un seul, mais deux ordres de contradictions éthiques dans nos sociétés. Il y a selon moi deux sources de la
${ }^{1}$ SPHERE CNRS-Université Paris Diderot, UMR 7219, bâtiment Condorcet, case 7093, 5, rue Thomas Mann, 75205 Paris Cedex 13, France.

${ }^{2}$ Rédacteur en chef de médecine/sciences, Équipe «Microenvironnement immunitaire et immunothérapie », Centre d'Immunologie et des Maladies Infectieuses (CIMI) - Inserm UMRS 1135 - Sorbonne Université, 91, boulevard de I'Hôpital, 75013 Paris, France. mariegaille@yahoo.fr jean-luc.teillaud@inserm.fr bioéthique, et celle qui passe au premier plan aujourd'hui (cela n'a pas toujours été le cas), ce sont des contradictions internes à la médecine. C'est ce que je veux dire en parlant de « deuxième âge de la bioéthique ». La médecine est une pratique, dans son fond, éthique, mais comme toute démarche humaine, également ambivalente. La médecine est éthique dans son principe, au sens où elle est portée par un souci de réparer des maux qui affectent les humains. C'est une démarche pour soigner, résoudre des problèmes du vivant humain. Une question est de savoir jusqu'où va cette démarche. Si on prend la définition de l'Organisation mondiale de la santé (OMS)l ${ }^{\text {, }}$

${ }^{1}$ https://www.who.int/about/mission/fr/ 
c'est très extensif. Mais en tout cas, la médecine, en ce qu'elle soigne des maux, est une démarche éthique.

Cela dit, la médecine rencontre de nombreuses contradictions. Elle peut comporter des violations d'une éthique, dans sa propre démarche éthique. On le sait au fond depuis Hippocrate ! Ces violations sont aussi la première source des procès de Nuremberg! De nouveaux maux peuvent surgir de la pratique qui vise à en éviter, par exemple, l'effet secondaire d'un médicament, le risque associé à un bénéfice. Il y a d'ailleurs une analyse bénéfice/ risque en médecine. Mais ce niveau-là n'est pas encore pour moi l'éthique médicale. La question surgit lorsque le médecin rencontre d'autres sources de mal et de souffrance que la maladie, et notamment la question du respect de la personne humaine, de la liberté et d'autres principes moraux et politiques. J'ai étudié d'assez près les textes du procès de Nuremberg, dont on dit à raison que c'est l'une des origines de la bioéthique; ils ajoutent une dimension spécifique aux autres crimes nazis, car les médecins ont essayé de plaider au nom de la recherche médicale. Et l'on peut penser aussi aux années 1960 aux États-Unis, quand s'élevaient des protestations contre certaines formes d'expérimentation ${ }^{2}$. On a affaire là à des contradictions qui sont aussi des contradictions entre acteurs : le médecin, le patient. La loi de 2002 sur les droits des patients est fondamentale sur ce plan. Le cœur de la bioéthique, selon moi, ce sont ici les contradictions entre les dimensions éthiques ; dans le soin lui-même, on veut soigner, mais on doit aussi respecter, et même le dévouement comporte le risque du pouvoir. Ce n'est pas une contradiction entre le bien et le mal, ce serait trop simple. Rien que dans la philosophie française, on a la pensée de ces deux extrêmes : la médecine du côté du bien, de la bienveillance, du souci de l'Autre, avec Emmanuel Lévinas ${ }^{3}$ comme figure tutélaire ; la médecine comme pouvoir, avec Michel Foucault ${ }^{4}$. Je pense que Lévinas et Foucault ont tous les deux raison et tort ; il y a une ambivalence et des tensions internes à la médecine. Et, aujourd'hui elles reviennent au premier plan.

Mais il y a une seconde source de contradictions éthiques, ce sont les contradictions entre des éthiques en un autre sens du terme, entre des représentations générales du monde et des systèmes de «valeurs »; elles surgissent, par exemple, quand une technique semble poser un problème dans la représentation de la vie humaine. Dans ce cas, on n'est plus seulement dans des contradictions entre des acteurs, mais entre des représentations de la vie. Par exemple, le CCNE français, le premier du genre dans le monde, a été créé à l'occasion du bébé éprouvette. Mais cela change notre représentation de la «procréation » (le terme même apparaît alors au premier plan).

Comment régler de tels dissensus ? Il y a eu une invention de l'État, qui consiste à dire : on n'est pas seulement dans un clivage politique droite/

\footnotetext{
${ }^{2}$ Henry K. Beecher. Ethics and clinical research. N EnglJ Med 1966 ; $274: 1354-60$.

${ }^{3}$ Lituanien d’origine, Emmanuel Lévinas (1906-1995) fut, très jeune, l'introducteur de la phénoménologie en France et penseur de l'altérité au travers de ses études talmudiques. Aujourd'hui, il est considéré comme l'un de nos plus prestigieux philosophes, célébré dans le monde entier. De Totalité et infini (La Haye, Martin Nijhoff, 1961) à Altérité et transcendance (Saint-Clément la Rivière, Fata Morgana, 1995), il a élaboré une éthique de l'altérité.

${ }^{4}$ Philosophe français, Michel Foucault (1926-1984) est mondialement connu pour ses analyses critiques de la psychiatrie, de la prison comme forme hégémonique du châtiment et des représentations de la sexualité. En lien avec le présent entretien, on peut lire La volonté de savoir - Histoire de la sexualité, tome 1 (Paris, Gallimard, 1976) et la retranscription de ses cours au Collège de France, notamment Le Pouvoir psychiatrique (1973-1974), Les Anormaux (1974-1975), Naissance de la biopolitique (1978-1979), tous publiés chez Gallimard $(2003,1999,2004)$.
}

gauche, on n'est pas seulement dans le rapport bénéfice/ risque, on ne peut se décider seulement en fonction de principes politiques; il faut aménager la discussion. Mais alors entre qui ? Des juristes, des médecins, mais aussi des représentants des «familles spirituelles et philosophiques », parce que la discussion engage ces représentations, ces valeurs. J'ai moi-même été recruté sur le contingent de membres qui relève de cette notion ( 5 sur les 40 très diverses sources de nomination) : en ce moment, je suis le « laïc » du CCNE (voir encart). J'ai sans doute été proposé à cause de mon travail sur le soin. Mais l'enjeu est réel : dans ce cas aussi, la bioéthique n'est pas une éthique, mais l'aménagement des contradictions éthiques, avec le risque de dire, comme je le disais plus haut, que cet aménagement soit purement procédural. La bioéthique est procédurale, elle ouvre un espace - ce terme d'espace, d'espace éthique, est essentiel, mais pas seulement. Pour moi, le plus important, est de savoir comment on décide et qui discute.

Avec la bioéthique, se constitue un débat, des positions, et peu à peu, par fragments, bouts, elle devient une éthique : non une éthique a priori, mais une construction progressive, issue de corpus hétérogènes, avec des concepts compliqués, voire bizarres: qu'est-ce que le don ? Qu'est-ce que le donneur décédé ? II y a des créations conceptuelles en bioéthique. C'est une construction démocratique, qui ne trouve sa source dans aucune tradition établie a priori : elle vient plutôt les bousculer, car elle les force à s'expliciter. Le rôle du philosophe dans cette affaire est assez surestimé dans ce groupe : il ne dépasse pas les contradictions, mais peut aider à les formuler. Mais cela ne suffit pas, il faut aussi s'engager. Il faut tous les regards, toutes les expertises, car toutes les contradictions de la société s'engouffrent dans la bioéthique. II y faudra la société civile et les patients. La question des experts est elle-même ambivalente: on ne peut pas s'en contenter, on ne peut pas s'en passer non plus. Ce serait démagogique de dire qu'on peut faire de l'éthique sans rien savoir du monde, des faits. C'est pour cela que si l'on ne peut s'arrêter à l'analyse bénéfice/ risque, ce niveau d'analyse demeure fondamental.

Dans ce deuxième âge de la bioéthique, on revient aux relations médicales, par distinction, donc, avec un premier âge dont l'enjeu était de concilier les religions et la laïcité dans un espace républicain. Cela reste très important aussi. Mais pour moi, le vrai problème que traite la bioéthique, ce ne sont pas ou pas seulement les divergences sur les essences (de la vie, la mort, etc.), ce sont les relations entre acteurs: par exemple, non la question de quoi (ce que l'on décide), mais de qui décide en fin de vie ou de la manipulation du génome dans le cas de pathologies d'origine monogénique; à quel moment la médecine va-t-elle 


\section{Le CCNE}

Le Comité Consultatif National d'Éthique pour les sciences de la vie et de la santé se compose d'un président, de 39 membres et de présidents d'honneur.

La loi assure au CCNE une pluridisciplinarité et un pluralisme qui permet de croiser les regards et les opinions sur chaque question. Le CCNE est composé de :

Un président, nommé par le président de la République pour une période de deux ans renouvelable. II conduit les travaux du CCNE.

39 membres nommés pour 4 ans :

- Cinq personnalités désignées par le président de la République appartenant aux «principales familles philosophiques et spirituelles » ${ }^{\star}$.

- 19 personnalités choisies pour «leur compétence et leur intérêt pour les problèmes éthiques »

- 15 personnalités appartenant au « secteur de la recherche » (Inserm, CNRS, Institut Pasteur, etc.)

Un secrétaire général coordonne les travaux du comité au quotidien.

* Article $4 \S 1^{\circ}$ du décret n $83-132$ du 23 février 1983 portant création d'un Comité consultatif national d'éthique pour les sciences de la vie et de la santé ; article $2 \S 1^{\circ}$ du décret relatif au Comité consultatif national d'éthique pour les sciences de la vie et de la santé, $n^{\circ}$ 97-555

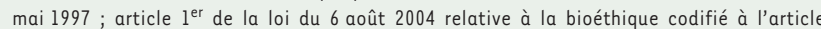
L 1412-2 $\S 1^{\circ} \mathrm{du}$ code de la santé publique.

trop loin, quelle différence y a-t-il entre le médical et le sociétal ? Ce sont plutôt des contradictions de la médecine que des contradictions entre des représentations. Cette distinction entre ces deux âges est à prendre en compte. Ces contradictions sont aussi inhérentes à la bioéthique française. Par exemple, la distinction entre le médical et le sociétal est très française. Elle est apparue à un moment pour traiter un certain type de problèmes et aujourd'hui, elle n'est peut-être pas dépassée totalement, mais la frontière se brouille. Sur la question de l'anonymat, un autre exemple : on a affaire à une technique qui introduit de la contradiction, avec la figure du père qui se démultiplie. L'anonymat est venu protéger la figure paternelle au sens juridique du terme de l'empiètement du donneur. L'anonymat est venu comme une construction pour régler celle-ci.

Ce constat n'empêche pas de reconnaître que sur la paternité, par exemple, il y a des questions anthropologiques sous-jacentes. Les deux âges de la bioéthique, aujourd'hui, sont désormais présents tous les deux. Ce n'est pas une succession strictement chronologique. On a plutôt des couches de débats : une couche un peu plus archaïque, avec le débat sur les valeurs ; et une autre, sociale, démocratique, réflexive, avec le débat sur les acteurs. Les deux sont intriquées. Personnellement, sur le fond, j'aurais tendance à traiter des questions bioéthiques comme des questions relationnelles et non d'essence : non « qu'est-ce que la vie? », « qu'est-ce qu'un enfant? », mais plutôt, quelles sont les relations sociales, médicales, morales, politiques et quelles sont leurs limites? Où commence ce qui peut les détruire, que je nomme des «violations »? II faut regarder du côté du négatif, sans doute le cœur de la bioéthique : toutes les variétés du négatif et du « mortel » dans la vie humaine, la maladie bien sûr, mais aussi la violence faite au citoyen, le conflit interne des valeurs jusqu'à la guerre civile. $\mathrm{m} / \mathrm{s}$. La rapidité des découvertes dans le domaine biomédical, leur traduction presque immédiate en possibilités thérapeutiques et en nouvelles pratiques de soins, l'introduction massive de l'intelligence artificielle dans le domaine biomédical, ne rendent-elles pas désormais très rapidement obsolète ce que le législateur français met en forme. Et alors que ces révolutions biomédicales s'effectuent à l'échelle mondiale, ne faudrait-il pas penser à d'autres temporalités législatives et dans un cadre qui ne soit pas seulement français, mais au moins européen?

FW. Je pense qu'il ne faut pas paniquer ! L'important est la construction et la solidité du cadre dont on parlait. II faut trouver le bon rythme, c'est sûr. Mais il faut prendre le temps de voir les seuils critiques qui sont franchis, avec leurs ambivalences propres; il faut aussi laisser les contradictions apparaître. Parfois malheureusement, celles-ci se manifestent via des cas, des conflits, des contentieux. II y a des choses compliquées qui apparaissent, qu'il faut suivre en direct, tout de suite. C'est notre rôle à tous. En revanche, pour moi, la loi a un autre rythme que celui de la science et de la bioéthique. Sur ce point, la bioéthique, de mon point de vue, se situe entre la loi et la science : elle doit être sur le terrain. La loi, elle, ne part jamais de zéro. On dispose de cadres, qui correspondent à beaucoup de choses. C'est aussi pour cela que chaque pays repart de sa tradition.

Quant à la globalisation des enjeux et au fait que des questions se posent de façon transnationale, hors des bornes de l'État-Nation, il ne faut pas se leurrer, le contexte international ouvre la possibilité à des fraudes, des abus, des transgressions. Cela ne constitue pas une raison pour renoncer à donner des cadres, au contraire. Mais il convient de ne pas dramatiser : il y a des cadres globaux, certes minimaux, mais existants, quelques interdits universels et il y aura, je pense, autour de l'environnement, par exemple, une construction globale progressive. Par ailleurs, il ne faut pas se polariser sur le mode de l'opposition entre barbarie et démocratie. En réalité, il y a des degrés, des paliers, des chemins différents empruntés par les démocraties pour aborder la même question. Dans ce contexte, la priorité est d'éviter la guerre civile, de préserver la confiance dans les institutions. Aujourd'hui, ce qu'on observe en France à travers la bioéthique, c'est une discussion qui a lieu sur des questions de vie ou de mort. En ce sens, elle est un espace de progrès de la démocratie. Le débat français est suivi, souvent avec ironie, mais il est très observé, car un tel débat y est possible. Personnellement, je cherche une double cohérence : théorique et pratique. Et je pense qu'il faut s'engager dans les institutions, parfois de manière critique, mais en leur sein. 
$\mathrm{m} / \mathbf{s}$. L'un des apports majeurs de votre travail philosophique est la pensée des moments (conjonctures/époques) : sommes-nous dans un moment caractérisable et remarquable du point de vue de l'éthique appliquée aux sciences biomédicales et aux pratiques cliniques, aux choix collectifs et individuels en matière de santé ?

FW. En effet, je pense qu'on est dans un moment particulier à l'heure actuelle, qui a un profond rapport avec la bioéthique. La bioéthique n'est pas une question parmi d'autres aujourd'hui, elle ne surgit pas par hasard au centre de la vie sociale et intellectuelle. Elle concentre tous les enjeux qui font que nous vivons, de mon point de vue, un moment historique nouveau, que je nomme le moment du vivant.

Un «moment », pour moi, est une sorte d'étape discontinue dans I'histoire de la pensée. Il y a des ruptures dans celle-ci, qui se traduisent par des convergences de certains problèmes, différents de ceux qui précédaient; il faut qu'il y ait convergence quasi-globale. Par exemple, si je prends les deux extrêmes de ce que je considère comme l'entrée dans un nouveau moment au début des années 1980 - L'homme neuronal de Jean-Pierre Changeux ${ }^{5}$ et les travaux de Michel Foucault sur la biopolitique, qui sont comme les deux pointes extrêmes d'un même compas - je pense qu'en réalité, ils nous disent tous les deux que la connaissance humaine et sur l'humain et la politique passent par celle du vivant. Et pour moi, tout a confirmé cette vision, ce basculement depuis le début des années 1980. II faut bien voir que ce basculement se traduit par des polémiques. Mais elles sont bien là, dès le départ ! Avant, on était dans un modèle dominé par une approche de sciences humaines, avec notamment la question du langage, de la culture. Réduire l'homme à un vivant, avant Changeux, Jonas, les théories du care, c'était une violence faite à l'humain. L'humain, c'était avant tout un être de culture, un vivant aussi, mais pas seulement, et sans doute pas primordialement. Pour moi, la bioéthique est la reconnaissance de ce moment, avec une complexité dans ce moment du vivant : on est des vivants, certes, mais de plusieurs façons. Avec Changeux et Foucault, on a l'idée que le vivant est ce qu'il faut penser, et chez Changeux, cette idée est assortie d'une tentation réductionniste mais aussi de perspectives d'élargissement du vivant, au social, à l'histoire, au travers de son exposition à divers environnements, par exemple.

Cependant, on a aussi l'idée, d'un autre côté, que nous sommes des objets de pouvoir sur le vivant. Est-il construit ou naturel ? C'est un faux problème, pour moi. En tout cas, nous sommes des êtres vivants complexes, avec des enjeux de pouvoir aussi bien que de connaissances. La bioéthique vient nous dire cela. Elle n'est pas par hasard au centre du moment présent. Elle est le point d'articulation entre la connaissance du vivant et le pouvoir sur le vivant. On a une vie humaine complexe, avec du savoir et du pouvoir, un peu des deux, et de la résistance - du soin, du soutien. La bioéthique est la clé de voûte du moment présent. Elle articule les deux éléments du vivant. La composition du CCNE renvoie à cela, avec des médecins, des biologistes, des philosophes, des juristes, etc. Les philosophes qui critiquent la bioéthique n'ont rien compris : ils confondent la bioéthique avec un moralisme. Ce n'est pas du tout cela !

\footnotetext{
${ }^{5}$ Neurobiologiste et généticien, président du CCNE de 1993 à 1999, ayant succédé à Jean Bernard.
}

Donc, j'appelle moment une reconfiguration d'ensemble de la vie humaine, un thème, un problème qui devient dominant. On est dans le tout bio : bio-pouvoir, biocatastrophe, bio-art, bio-savoir, etc. Le vivant devient notre boussole, mais pour le meilleur et pour le pire.

Ce que j'ai fait en philosophie française du XXe siècle, cette approche par les moments, a convergé vers ma préoccupation pour la bioéthique contemporaine. II faut cependant s'inquiéter d'un point qui devient critique : ce moment n'a pas pris tout de suite, et pas encore complètement, conscience de lui-même. Nos générations ont vécu ce moment sans le savoir. Il a manqué à ce moment la réflexivité critique, peut-être parce que le moment antérieur était très puissant. Maintenant, tout le monde se réveille, s'intéresse au cerveau, à l'environnement, à la biologie. Dans ma génération, c'est très clair. Je suis né en 1964, en 1983, paraît L'homme neuronal, jugé comme très réductionniste. Aujourd'hui, on nous dit que l'homme est un animal comme les autres, fin de l'exception humaine! Cela me semble absurde d'ailleurs. Je suis plus proche de positions comme celles d'Alain Prochiantz ${ }^{6}$ : l'homme reste un être vivant un peu spécifique parmi les autres vivants, notamment en raison du langage. Même si le langage est intégralement une fonction biologique, il change encore un peu quelque chose.

Pour revenir à la question de la réflexivité, c'est troublant, car les autres moments que j'ai étudiés l'étaient, réflexifs : l'existentialisme, le structuralisme se sont pensés comme moments, en rupture. Nous, on est allé en ordre dispersé, on n'a pas compris qu'on rentrait dans une autre époque. Foucault l'avait compris, mais sur un mode seulement critique. II ne faut pas seulement critiquer. II faut aussi accompagner, entrer un peu dans les sciences du vivant. De mon côté, j'ai beaucoup travaillé sur les théories de l'attachement. On peut penser aussi au travail de Catherine Malabou, philosophe ${ }^{7}$. Je ne suis pas du tout du côté des philosophies critiques de la biologie, des philosophies radicalement anti-scientifiques. La composition du CCNE pour moi fait sens, même si elle est imparfaite, dans l'idée qu'on va non pas échapper au pouvoir sur le vivant, mais le réguler un peu. Cela me semble être la bonne attitude. II faut peut-être le recul historique pour s'en rendre compte et c'est pour cela que j'assume le lien entre mon approche en histoire de la philosophie et mon engagement dans la bioéthique.

${ }^{6}$ Alain Prochiantz est un chercheur en neurobiologie et professeur au Collège de France dont il est devenu l'administrateur en 2015.

${ }^{7}$ Catherine Malabou est professeure de philosophie au Center for Research in Modern European Philosophy (université de Kingston, Royaume-Uni), et également professeure d'études européennes à l'Université de Californie à Irvine (États-Unis). 
$\mathrm{m} / \mathrm{s}$. Un autre élément constitutif de votre œuvre est la pensée du soin: qu'est-ce qui fait que nous prenons soin, ou pas? Quels sont les différentes facettes du soin?

FW. Mon intuition philosophique personnelle, qui ne vient pas de Bergson en tant que tel, est celle de l'émergence de la morale et de la politique depuis les relations entre les vivants humains. Comment émergent des normes, depuis le vivant (mais pas seulement dans chaque vivant, entre eux aussi, d'où la limite de ma proximité avec Georges Canguilhem ${ }^{8}$, ou plutôt le prolongement que je voudrais apporter à sa pensée). Je suis venu au soin par la violation des relations vitales: le soin est une réponse à un risque biologique ou plutôt vital, moral, et social à la fois. C'est pour cela que je m'intéresse à la théorie de l'attachement, sur la construction du concept de l'attachement en psychologie expérimentale, les normes de l'attachement - sécure, insécure, etc.

Je pense qu'il y a deux aspects dans l'attachement, les modules cérébraux, mais aussi les aventures de l'attachement, l'attachement à la personne qu'on a sous les yeux quand on est nourrisson. À cet égard, le lien entre psychanalyse et biologie est devenu peu à peu très important pour moi, mais surtout la question de la négativité dans le vivant: le soin, c’est la réponse à la négativité vitale, non seulement pathologique, individuelle, mais aussi morale et interindividuelle. La morale naît du refus de la négativité entre les vivants : la maladie, la violence. Car au fond, cela ne suffit pas de dire que le médecin vient soigner le malade, que la mère vient s'occuper du nourrisson. En fait, pourquoi la mère vient s'occuper du nourrisson? Pourquoi le médecin soigne le malade? II peut ne venir personne aussi. C'est la question philosophique ultime pour moi. L'attachement lui-même peut être clos, être un facteur de guerre: mes enfants contre les vôtres. Mais quand l'attachement est suffisamment bien réussi, comme le montre Donald Woods Winnicott ${ }^{9}$, il y a une ouverture possible sur le monde. II y a deux pathologies de l'attachement: la détresse, qui fait qu'on ne s'attache à personne; l'attachement excessif, fusionnel, qui fait que la personne ne peut s'ouvrir sur le monde. Le nationalisme pour moi est une pathologie de l'attachement. II exprime la peur de l'autre, mais en fait l'absence - non métaphorique - de confiance en soi, d'amour. Ma boussole est donc bien la question de la négativité, dans le vivant et de ses diverses dimensions, notamment entre les humains, puis dans leur rapport aux autres vivants et au monde.

$\mathrm{m} / \mathrm{s}$. En 2012, vous avez publié un livre personnel autant que philosophique, Revivre - Éprouver nos blessures et nos ressources. On retrouve

\footnotetext{
${ }^{8}$ Georges Canguilhem (1904-1995) est un philosophe français, également médecin de formation. Spécialiste d'épistémologie et d'histoire des sciences, il publia des ouvrages très importants sur la constitution de la biologie comme science, sur la médecine, la psychologie, les « idéologies scientifiques » et l'éthique. Sa thèse de médecine est devenue un classique de la philosophie : Le normal et le pathologique (Paris, PUF, 1966). II a publié de nombreux recueils d'articles d'histoire et de philosophie des sciences du vivant, notamment la biologie, et sur la médecine, notamment Écrits sur la médecine (Paris, Seuil, 2002). La publication de ses œuvres complètes est en cours aux éditions Vrin.

${ }^{9}$ Donald Woods Winnicott (1896-1971), pédopsychiatre et psychanalyste britannique, spécialiste du développement de l'enfant et également connu pour sa théorie du faux «self ». On pourra notamment lire de lui La famille suffisamment bonne (Paris, Payot, 2010), La mère suffisamment bonne (Paris, Payot, 2006), De la pédiatrie à la psychanalyse (Paris, Payot, 1989), Processus de maturation chez l'enfant (Paris, Payot, 1988).
}

dans ce titre votre dialogue avec Winnicott et son attention à la personne, à son parcours propre, ses failles mais aussi ses rebonds. Comment selon vous une loi de bioéthique, qui porte sur les aspects cruciaux de l'existence humaine, peut-elle faire place à une telle attention?

FW. Le « revivre » a deux sens : celui de la répétition négative et celui de la renaissance. Quel est le lien entre ces deux sens? C'est le cœur de nos vies, de leur ambivalence et de leur double sens. De même, dans les maladies chroniques, il y a une violence intérieure indépassable, le corps « contient» ce risque (la maladie devient comme un corps étranger), mais la médecine peut le « contenir » en un tout autre sens, opposé et qui permet de vivre. II faut lutter contre, ce n'est jamais parfait. II n'y a pas seulement un ennemi de l'extérieur. La chronicité, en outre, ce n'est pas du tout l'idée que la chose est permanente, c'est le contraire : l'idée de variation, et de maintenir en dessous d'un seuil, celui de l'aigu dans la maladie, qui peut être mortel. Et c'est vrai en politique.

Pour moi, il y a deux illusions : croire qu'on est toujours sain, et comme société, qu'il n'y a pas, par exemple, de fascistes ; croire à l'inverse qu'on ne peut rien faire, qu'on ne peut pas contenir le mal. La démocratie électorale est un palier du traitement, insuffisant et fragile, mais réel. Mais pour contenir le chronique, il faut des progrès. Quand on lit le témoignage des malades chroniques, on voit qu'il ne faut pas seulement lutter contre la maladie, mais aussi jouir de la vie, être heureux. On ne lutte contre la régression qu'en progressant. On peut franchir des paliers et à chaque fois, on franchit des seuils absolus en évitant des régressions.

\section{$\mathrm{m} / \mathrm{s}$. Le CCNE doit-il étendre son domaine?}

FW. On a publié des avis sur beaucoup de sujets, la biodiversité, les migrants. Il y a des dimensions transversales: le numérique, l'économie, l'environnement, l'international. Doit-on rester spécifique ou devenir une instance éthique en général ? Ma position est qu'on doit être à la fois spécifiques et globaux à travers cet angle spécifique. Cela tient à ma position sur le moment. La santé n'est pas, n'est plus un problème local. Elle est un angle spécifique avec des enjeux globaux.

Ce débat présuppose certes l'essentiel, qui tient aussi à la démocratie, sur les sujets brûlants de nos vies, c'est une avancée pour la démocratie, mais aussi pour la vie: si l'on peut se poser la question de cette extension, cela veut dire qu'on a une forme de confiance dans le débat. Le CCNE a acquis une sorte de légitimité. C'est une institution démocratique vivante qu'il faut absolument protéger. Mais je reste favorable à une focale placée sur le 
vivant, contre les critiques qui ne comprennent pas que la bioéthique n'est pas une morale (trop faible ou trop forte, selon les goûts), ni trop ni trop peu de morale, mais une réflexion et un aménagement des contradictions entre les morales. Elle est une façon d'accueillir toutes les négativités du vivant (vitales, morales, sociales, politiques).

Je souhaiterais proposer une approche complète de la vie et de la mort : un vitalisme critique. La même chose se produit à mon avis sur l'environnement : ça se construit peu à peu, à travers les conférences des parties (COP), on ne peut pas faire autrement, on n'a plus le choix. II ne se passera peut-être rien. Mais mon espoir est placé dans tous les acteurs de la société, les journalistes, les lanceurs d'alertes, tous les contre-pouvoirs, et les citoyens, les médiations, les institutions, qui ne voudront pas que certaines choses qui relèvent non seulement du monde, mais des relations entre les humains, se traduisent dans leurs corps et les détruisent. $\diamond$

\section{Meeting with Frédéric Worms}

Philosopher and professor of contemporary

philosophy at the "École Normale Supérieure", member of the National ethics advisory committee

\section{for life sciences and health}

\section{LIENS D'INTÉRÊT}

Les auteurs déclarent n'avoir aucun lien d'intérêt concernant les données publiées dans cet article.

\section{TIRÉS À PART}

M. Gaille

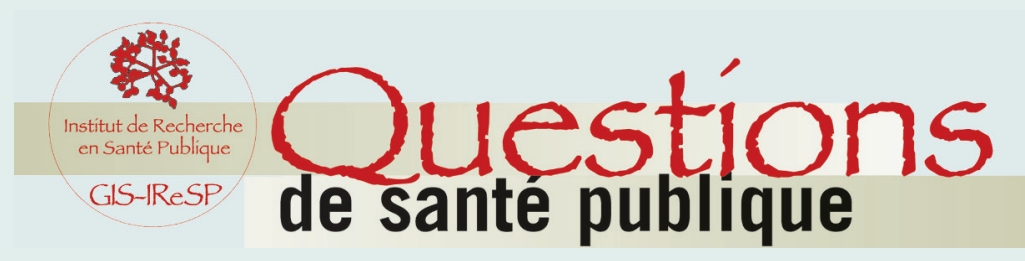

\section{Un nouveau bulletin}

pour une meilleure visibilité des résultats

de la recherche en santé publique

es résultats de la recherche en santé publique public concerné) les informations les plus récentes souffrent en France d'un réel manque de visibilité. Ceci concerne aussi bien le monde académique (hors santé publique) que le grand public et les décideurs. Pour pallier ce déficit, l'IReSP a créé un bulletin à large diffusion intitulé "Questions de santé publique ", largement inspiré du bulletin mensuel d'information de I'INED " Populations et sociétés ". L'objectif éditorial est de porter à la connaissance d'un large public (enseignants, étudiants, journalistes, décideurs, milieux de la recherche, associations, concernant des questions importantes de santé publique, rédigées de façon facilement lisible et compréhensible pour des non spécialistes, en garantissant que les informations publiées sont validées scientifiquement. La publication concerne des faits et non des positions. Audelà de la présentation de résultats, les qualités pédagogiques de Questions de santé publique permettent au lecteur de mieux comprendre comment sont formulées et abordées les questions de santé publique et quelles sont les limites de ces études.

Nom

Prénom

Institution

Fonction

Spécialité

Service

Adresse

Ville

Code postal

Pays

Adresse électronique

à nous retourner par la poste ou par fax au 0149850345

Questions de santé publique

EDP Sciences

17 avenue du Hoggar

91944 Les Ulis

France

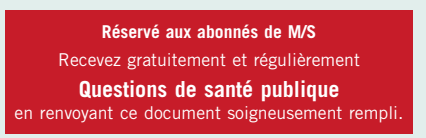

Questions de santé publique est une publication de l'Institut de Recherche en Santé Publique. I Directeur de la publication : Corinne Alberti. I Comité de rédaction : Sarah Bellouze, Marion Cipriano, Alexandre Cobigo et Jean-Marie Gagliolo. I Réalisation : EDP Sciences. 